\title{
Predictors for advanced liver fibrosis in chronic hepatitis B virus infection with persistently normal or mildly elevated alanine aminotransferase
}

\author{
DEXIN WANG, PING ZHANG and MIN ZHANG \\ Department of Medicine, The Sixth People's Hospital of Qingdao, Qingdao, Shandong 266033, P.R. China
}

Received February 14, 2017; Accepted July 27, 2017

DOI: $10.3892 /$ etm.2017.5219

\begin{abstract}
The aim of the present study was to evaluate the predictors for advanced liver fibrosis in patients with chronic hepatitis B virus (HBV) infection with persistently normal alanine aminotransferase (PNALT), or persistently or intermittently mildly elevated ALT (PIEALT). A total of 305 patients were included in the present study. Liver biopsies were evaluated using the METAVIR scoring system. Liver stiffness (LS) was measured using Fibroscan. Multivariate logistic regression and the area under the receiver operating characteristic curve (AUROC) were used to examine the diagnostic value of the predictors for advanced liver fibrosis. HBV DNA viral load in the PNALT group was significantly lower compared with the

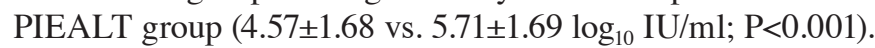
Body mass index and LS were also significantly lower in the PNALT group compared with the PIEALT group $(\mathrm{P}<0.001)$. The proportion of patients with liver fibrosis was significantly higher in the PIEALT group compared with the PNATL group $(\mathrm{P}=0.001)$. High ALT levels were an independent predictor for liver fibrosis, with an odds ratio $(\mathrm{OR})$ of $2.69(\mathrm{P}=0.002)$. Male sex $(\mathrm{OR}=0.34, \mathrm{P}=0.007)$, high ALT levels $(\mathrm{OR}=2.37, \mathrm{P}=0.029)$ and a high HBV DNA load $(\mathrm{OR}=1.39, \mathrm{P}=0.005)$ were independent predictors for advanced liver fibrosis. The AUROC was $0.65(\mathrm{P}=0.003)$ when using ALT levels to predict advanced liver fibrosis. ALT levels at $\geq 0.88$ upper limit of normal (ULN; $35 \mathrm{IU} / \mathrm{l})$ were considered as positive for advanced liver fibrosis, the sensitivity and specificity were 87.8 and $47.4 \%$, respectively. The AUROC was $0.64(\mathrm{P}=0.004)$ when using the HBV
\end{abstract}

Correspondence to: Dr Min Zhang, Department of Medicine, The Sixth People's Hospital of Qingdao, 9 Fushun Road, Qingdao, Shandong 266033, P.R. China

E-mail: xhkzhangmin@126.com

Abbreviations: ALT, alanine aminotransferase; CHB, chronic hepatitis B; HCC, hepatocellular carcinoma; HBV, hepatitis B virus; LS, liver stiffness; TE, transient elastography; ULN, upper limit of normal

Key words: hepatitis B, liver fibrosis, METAVIR scoring system, Fibroscan, alanine aminotransferase, upper limit of normal
DNA value to predict advanced liver fibrosis. When an HBV DNA value of $\geq 4.99 \log _{10} \mathrm{IU} / \mathrm{ml}$ was considered as positive for advanced liver fibrosis, the sensitivity and specificity were 78.0 and $49.5 \%$, respectively. The AUROC was $0.72(\mathrm{P}<0.001)$ when combining ALT, HBV DNA load and sex into a formulation to predict advanced liver fibrosis. When the formulation score at $>-2.22$ was considered as positive for advanced liver fibrosis, the sensitivity and specificity were 61.5 and $70.7 \%$, respectively. Therefore, normal ALT levels do not always indicate the absence of hepatic fibrosis. A combination of ALT levels, sex and serum HBV DNA load may more effectively identify patients with $\mathrm{CHB}$ at high risk of developing fibrosis. These patients may benefit from liver biopsy.

\section{Introduction}

It is estimated that $\sim 400$ million people worldwide are chronically infected with hepatitis B virus (HBV) $(1,2)$. Patients with chronic hepatitis $\mathrm{B}(\mathrm{CHB})$ are at increased risk of developing end-stage liver diseases, including liver cirrhosis, liver failure and hepatocellular carcinoma (HCC) (3). Therefore, the goal of anti-HBV treatment is to suppress HBV replication in a sustained manner, to achieve hepatitis $B$ e antigen (HBeAg) sero-conversion among patients with $\mathrm{CHB}$ that are $\mathrm{HBeAg}$ sero-positive, and to the improve quality of life and survival of patients by preventing progression to end-stage liver disease (4).

The progression of chronic HBV infection has been determined and divided into three phases, consisting of the immune tolerant, immune clearance and residual or inactive phases. This division is based on HBV DNA viral load, HBeAg and serum levels of alanine aminotransferase (ALT) (4). Patients with CHB in the immune tolerant phase are usually young and HBeAg sero-positive with a high HBV DNA load, but have persistently normal ALT levels (PNALT). During the immune clearance phase, patients with $\mathrm{CHB}$ often exhibit elevated serum levels of ALT, complicated by hepatic decompensation in certain cases (5). ALT elevation is considered to be the result of the host immune response against $\mathrm{HBV}$-infected hepatocytes (6). Higher ALT levels, therefore, usually reflect more extensive hepatocyte damage due to an increased immune response, which leads to liver fibrosis and marked disease progression. Clinical CHB management guidelines suggest that antiviral treatment should be commenced if 
patients exhibit persistently elevated ALT levels, defined as $>2$ times the upper limit of normal (ULN), for at least one month between observations (4). For patients with PNALT or persistently or intermittently mildly elevated ALT (PIEALT), no drug treatments are recommended unless they exhibit symptoms of advanced fibrosis or liver cirrhosis (5).

Monitoring the progress of liver fibrosis in patients remains clinically challenging. Currently, liver biopsy is the gold standard for evaluating liver fibrosis (7). However, liver biopsy has also been criticized as it leads to sampling errors, underestimation of liver fibrosis and potentially severe complications (8). It is also unrealistic to perform liver biopsies on a large population. Therefore, it is important to identify patients with $\mathrm{CHB}$ and PNALT at high risk of developing liver fibrosis. However, the current predictors of liver fibrosis, such as age, sex, serum ALT level and HBV DNA viral load, in patients with CHB and PNALT or mildly elevated ALT are limited $(9,10)$.

In the present study, liver biopsies were performed in patients with CHB and PNALT or PIEALT in order to explore the predictors for liver fibrosis in patients with $\mathrm{CHB}$ and PNALT or PIEALT.

\section{Materials and methods}

Patients. A total of 305 patients with CHB were analyzed in the present study. The patients were treated and enrolled between February 2011 and September 2015 in the Department of Medicine, The Sixth People's Hospital of Qingdao (Qingdao, China). The Institutional Review Board of the Sixth People's Hospital of Qingdao approved the study and all patients provided written informed consent prior to enrollment. Chronic $\mathrm{HBV}$ infection was defined as sero-positive for hepatitis B surface antigen (HBsAg) for $\geq 6$ months (5). The inclusion criteria were as follows: i) Diagnosis with chronic HBV infection and ii) ALT values $<80$ IU/1 on $\geq 3$ separate occasions ( $\geq 1$ month between each observation). The exclusion criteria were as follows: i) The presence of hepatitis $\mathrm{C}$ or D, or human immunodeficiency virus co-infection; ii) medical evidence of liver disease due to alternative etiology; iii) use of hepatotoxic drugs or heavy alcohol consumption (>30 g/day) (11); iv) patient had already received antiviral therapy or liver protectant reagents and v) ALT levels detected $<3$ times prior to liver biopsy.

Patient evaluation. Patient information, including age, sex and body mass index (BMI) was recorded. Serum HBV DNA viral load, serum HBV markers and ALT levels were measured in all patients. Patients who presented with liver fibrosis [liver stiffness (LS) >6.35 kPa] underwent liver biopsy (9). ALT and aspartate transaminase (AST) levels were determined using the Olympus AU5400 biochemical analyzer (Olympus Corporation, Tokyo, Japan). The ULN for ALT was $40 \mathrm{U} / 1$. Patients were categorized as PIEALT if they had $\geq 3$ ALT values ( $\geq 1$ month between each observation) $<80 \mathrm{IU} / 1$, and $\geq 1$ ALT value of $>40$ IU/l in the previous 6 months. Patients were categorized as PNALT if they had $\geq 3$ ALT values $<40$ IU/1 over the previous 6 months.

Evaluation of $H B V$ markers. HBsAg (cat no. 6C36-44), HBsAb (cat no. 7C18-25), HBeAg (cat no. 6C32-25), HBeAb (cat no. 6C34-20) and $\mathrm{HBcAb}$ (cat no. 8L44-25) were detected using Abbott ELISA kits (Roche Molecular Diagnostics, Pleasanton, CA, USA.).

Evaluation of serum HBV DNA levels. Serum HBV DNA levels were measured by quantitative polymerase chain reaction, using the Cobas Ampliprep/Cobas TaqMan, version 2.0 (Roche Molecular Diagnostics), according to the manufacturer's protocol. The manufacturer reports an HBV DNA linear range of 20 to $1.7 \times 10^{8} \mathrm{IU} / \mathrm{ml}(1 \mathrm{IU} / \mathrm{ml}=5.82 \mathrm{copies} / \mathrm{ml})(12)$.

Liver biopsy. Liver biopsy was performed using a 16-gauge Menghini biopsy needle (Aspen Medical Europe, Ltd., Ashby-de-la-Zouch, UK). For diagnosis, $\geq 20 \mathrm{~mm}$ liver tissue was obtained (13). All liver tissues were fixed at $4 \%$ paraformaldehyde in $0.1 \mathrm{M}$ phosphate buffer for $24 \mathrm{~h}$ and embedded in paraffin wax. Then the paraffin-embedded sample were sliced into $4-\mu \mathrm{m}$ sections and mounted onto glass slides. After dewaxing, the slide was dipped into a Coplin jar containing Mayer's hematoxylin and agitated for $30 \mathrm{sec}$. Then the slide was dipped into $1 \%$ eosin Y solution for 10-30 sec with agitation. The sections were dehydrated with two changes of $95 \%$ alcohol and two changes of $100 \%$ alcohol for 30 sec each. The alcohol was extracted with two washes of xylene. Slides were observed under a light microscope. Liver fibrosis was staged according to the METAVIR scoring system as follows: No fibrosis (F0), mild fibrosis (portal fibrosis without septa, F1), moderate fibrosis (portal fibrosis with few septa, F2), severe fibrosis (numerous septa without cirrhosis, F3) or cirrhosis (F4). The scores were determined by a liver pathologist without knowledge of LS value (14). Liver fibrosis was diagnosed when METAVIR score $\geq \mathrm{F} 1$ and advanced liver fibrosis was diagnosed when METAVIR score $\geq F 2$.

Evaluation of LS. Fibroscan, also known as transient elastography (TE), was performed by three trained operators, following the manufacturer's protocol. The operators used Fibroscan (FibroScan ${ }^{\circledR} 502$ Touch system; Echosens, Paris, France) equipped with a standard probe. The procedure was performed as previously described (15). A result was considered reliable if: i) $\geq 10$ successful shots of TE; ii) the success rate was $\geq 60 \%$ and iii) the inter-quartile median ratio was $<30 \%$. The results of TE are expressed in $\mathrm{kPa}$.

Statistical analysis. The measurement units are expressed as the mean \pm standard deviation for normally distributed data and median (range) for data with a non-normal distribution. Categorical data are expressed as percentages. Univariate and multivariate analyses were used to evaluate the predictors of liver fibrosis and advanced liver fibrosis. The $\chi$-squared test was applied to compare liver fibrosis METAVIR scores. To determine the performance of ALT and HBV DNA in predicting clinical outcomes, the area under the receiver operating characteristic curve (AUROC) and logistic regression were calculated. This was also performed for a combined formulation of ALT and HBV DNA, according to the following formula: $-2.632+(0.93 \times$ ALT $)+(0.258 \times$ DNA $)-(0.995 \times$ sex $)$ (male $=1$, female $=2)$. All analyses were performed using SPSS version 13.0 (SPSS, Inc., Chicago, IL, USA). P<0.05 was considered to indicate a statistically significant difference. 
Table I. Demographics and clinical characteristics.

\begin{tabular}{lccr}
\hline Characteristics & PNALT group $(\mathrm{n}=104)$ & PIEALT group $(\mathrm{n}=201)$ & P-values \\
\hline Sex, male $(\%)$ & $49(47.1)$ & $104(51.7)$ & 0.444 \\
Age, years & $33.48 \pm 9.31$ & $34.03 \pm 9.18$ & 0.616 \\
HBeAg, positive $(\%)$ & $41(39.4)$ & $97(48.3)$ & 0.142 \\
HBV DNA, $\log _{10} \mathrm{IU} / \mathrm{ml}$ & $4.57 \pm 1.68$ & $5.71 \pm 1.69$ & $<0.001$ \\
BMI & $21.44 \pm 2.53$ & $23.06 \pm 3.65$ & $<0.001$ \\
LS, kPa & $7.22 \pm 1.41$ & $8.34 \pm 2.37$ & $<0.001$
\end{tabular}

Data are presented as the mean \pm standard deviation or number (percentage). ALT, alanine aminotransferase; PNALT, persistently normal ALT; PIEALT, persistently or intermittently mildly elevated ALT; HBeAg, hepatitis B e antigen; HBV, hepatitis B virus; BMI, body mass index; LS, liver stiffness.

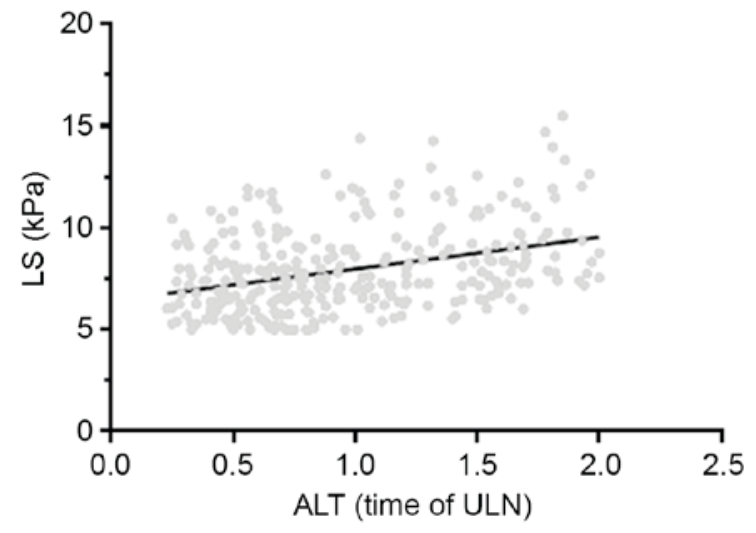

Figure 1. Association between ALT levels and LS in patients with chronic hepatitis B $(n=305)$. ALT levels were positively correlated with LS $(r=0.34$, $\mathrm{P}<0.001)$. ALT, alanine aminotransferase; ULN, upper limit of normal; LS, liver stiffness.

\section{Results}

Demographics and clinical characteristics. A total of 305 patients with $\mathrm{CHB}$ were included in the current study (Table I). Of these, there were 104 patients in the PNALT group with a mean age of $33.48 \pm 9.31$ years. There were 201 patients in the PIEALT group with a mean age of $34.03 \pm 9.18$ years. There were $49(47.1 \%)$ and 104 males $(51.7 \%)$ in the PNALT and PIEALT groups, respectively. There were no significant differences between the sex and ages of the patients in the two groups. However, HBV DNA viral load in the PNALT group was significantly lower compared with the PIEALT group $\left(4.57 \pm 1.68 \log _{10}\right.$ vs. $5.71 \pm 1.69 \log _{10} \mathrm{IU} / \mathrm{ml}$; $\mathrm{P}<0.001)$. BMI was significantly lower in the PNALT group compared with the PIEALT group (21.44 \pm 2.53 vs. 23.06 \pm 3.65 ; $\mathrm{P}<0.001)$. LS measured by TE was significantly higher in the PIEALT group compared with the PNALT group $(8.34 \pm 2.37$ vs. $7.22 \pm 1.41 \mathrm{kPa} ; \mathrm{P}<0.001)$.

Evaluation of the association between sex, ALT levels and LS. ALT levels in the 305 patients were positively correlated with the LS value ( $\mathrm{r}=0.34, \mathrm{P}<0.001$; Fig. 1$)$. The association between sex and LS was evaluated (Fig. 2). No significant differences in LS values were observed between male and females in the

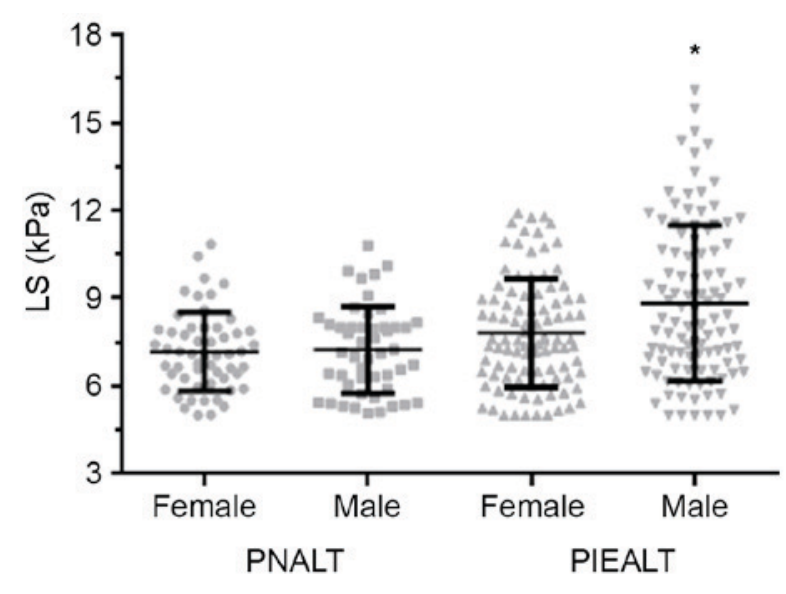

Figure 2. Association between sex and LS in patients with chronic hepatitis B LS values were compared between male and female patients with PNALT ( $n=49$ and $n=55$, respectively) and between male and female patients with PIEALT ( $n=104$ and $n=97$, respectively). ${ }^{*} \mathrm{P}<0.01$ vs. female PIEALT group. PNALT, persistently normal ALT; PIEALT, persistently or intermittently mildly elevated ALT; ALT, alanine aminotransferase; LS, liver stiffness.

PNALT group $(7.24 \pm 1.48$ vs. $7.18 \pm 1.34 \mathrm{kPa})$. However, in the PIEALT group, males exhibited a significantly higher LS value compared with females $(8.83 \pm 2.66$ vs. $7.82 \pm 1.87 \mathrm{kPa} ; \mathrm{P}<0.01)$.

Analysis of predictors for liver fibrosis among patients with PNALT and PIEALT. A total of 74 and 159 patients with LS $>6.35 \mathrm{kPa}$ were identified in the PNALT and PIEALT groups, respectively. Liver biopsy was performed in these patients. Among the 74 patients in the PNALT group, 34 patients had a METAVIR fibrosis score of F0, 38 patients had a METAVIR score of $\mathrm{F} 1$ and 2 patients had a METAVIR score of F2. Among the 159 patients with LS $>6.35 \mathrm{kPa}$ in the PIEALT group, 39 patients had a METAVIR fibrosis score of $\geq \mathrm{F} 2$. A total of $54.1 \%(40 / 74)$ and $72.3 \%(115 / 159)$ of patients with PNALT or PIEALT had liver fibrosis (METAVIR score $\geq F 1$ ), respectively. The proportion of patients with liver fibrosis was significantly higher in the PIEALT group compared with the PNALT group $(\mathrm{P}<0.001$; Table II).

A multivariate logistic regression analysis was performed for predictors of liver fibrosis (Table III) and advanced liver fibrosis (Table IV). Among patients with PNALT and PIEALT, high ALT levels was the only strong predictor for liver fibrosis 
Table II. Proportion of patients with liver fibrosis based on the METAVIR scoring system.

\begin{tabular}{lcc}
\hline Variable & PNALT group & PIEALT group \\
\hline Liver biopsy, $\mathrm{n}$ & 74 & 159 \\
Liver biopsy result, $\mathrm{n}(\%)$ & & \\
F0 & $34(45.9)$ & $44(27.7)$ \\
F1 & $38(51.4)$ & $76(47.8)$ \\
F2 & $2(2.7)$ & $35(22.0)$ \\
F3 & $0(0)$ & $3(1.9)$ \\
F4 & $0(0)$ & $1(0.6)$ \\
Liver stiffness, $(\mathrm{kPa})$ & $7.86 \pm 1.12$ & $9.07 \pm 2.12$ \\
\hline
\end{tabular}

Data are presented as the mean \pm standard deviation or number (percentage). ALT, alanine aminotransferase; PNALT, persistently normal ALT; PIEALT, persistently or intermittently mildly elevated ALT.

Table III. Univariate and multivariate logistic regression analysis of predictors for liver fibrosis.

\begin{tabular}{|c|c|c|c|c|c|c|}
\hline \multirow[b]{2}{*}{ Variable } & \multicolumn{3}{|c|}{ Univariate analysis } & \multicolumn{3}{|c|}{ Multivariate analysis } \\
\hline & OR & $95 \% \mathrm{CI}$ & $\mathrm{P}$-value & OR & $95 \% \mathrm{CI}$ & P-value \\
\hline Sex & 0.57 & $0.33-0.99$ & 0.043 & 0.57 & $0.32-1.01$ & 0.052 \\
\hline Age (years) & 0.74 & $0.97-1.03$ & 0.739 & & & \\
\hline ALT levels & 2.41 & $1.33-4.38$ & 0.004 & 2.69 & $1.39-5.18$ & 0.002 \\
\hline HBV DNA & 1.12 & $0.97-1.31$ & 0.134 & & & \\
\hline BMI & 1.04 & $0.95-1.13$ & 0.392 & & & \\
\hline AST levels & 1.17 & $0.49-2.75$ & 0.724 & & & \\
\hline HBeAg status & 0.63 & $0.36-1.10$ & 0.105 & & & \\
\hline
\end{tabular}

OR, odds ratio; CI, confidence intervals; ALT, alanine aminotransferase; HBV, hepatitis B virus; AST, aspartate aminotransferase; BMI, body mass index; $\mathrm{HBeAg}$, hepatitis B e antigen.

Table IV. Univariate and multivariate logistic regression analysis of predictors for advanced liver fibrosis.

\begin{tabular}{lccccr}
\hline & \multicolumn{3}{c}{ Univariate analysis } & & \multicolumn{2}{c}{ Multivariate analysis } \\
\cline { 2 - 5 } Variable & OR & $95 \%$ CI & P-value & OR & $95 \%$ CI \\
\hline Sex & 0.37 & $0.18-0.77$ & 0.008 & 0.34 & $0.15-0.75$ \\
Age (years) & 1.01 & $0.98-1.05$ & 0.532 & & 0.007 \\
ALT levels & 2.89 & $1.42-5.88$ & 0.003 & 2.37 & $1.09-5.15$ \\
HBV DNA & 1.35 & $1.09-1.66$ & 0.004 & 1.39 & 0.029 \\
BMI & 1.06 & $0.96-1.18$ & 0.237 & & 0.005 \\
AST levels & 3.41 & $1.28-9.09$ & 0.014 & 2.07 & $0.68-6.28$ \\
HBeAg status & 0.86 & $0.44-1.69$ & 0.670 & & 0.200 \\
\hline
\end{tabular}

OR, odds ratio; CI, confidence intervals; ALT, alanine aminotransferase; HBV, hepatitis B virus; AST, aspartate aminotransferase; BMI, body mass index; $\mathrm{HBeAg}$, hepatitis B e antigen.

with an odds ratio $(\mathrm{OR})$ of $2.69(\mathrm{P}=0.002)$ (Table III). However, male sex $(\mathrm{P}=0.007)$, high ALT levels $(\mathrm{P}=0.029)$ and high HBV DNA load $(\mathrm{P}=0.005)$ were all independent predictors for advanced liver fibrosis (Table IV).
Patients were further stratified according to HBeAg status for regression analysis (Table $\mathrm{V}$ ). Among patients that were HBeAg positive, AST levels $(\mathrm{P}=0.049)$, sex $(\mathrm{P}=0.009)$ and HBV DNA viral load $(\mathrm{P}=0.041)$ were independent predictors 
Table V. Univariate and multivariate logistic regression analysis of predictors for advanced liver fibrosis in patients with positive or negative $\mathrm{HBeAg}$ status.

A, HBeAg positive patients $(n=138)$

\begin{tabular}{|c|c|c|c|c|c|c|}
\hline \multirow[b]{2}{*}{ Variables } & \multicolumn{3}{|c|}{ Univariate analysis } & \multicolumn{3}{|c|}{ Multivariate analysis } \\
\hline & OR & $95 \% \mathrm{CI}$ & P-value & OR & $95 \% \mathrm{CI}$ & P-value \\
\hline Sex & 0.22 & $0.07-0.72$ & 0.012 & 0.18 & $0.05-0.66$ & 0.009 \\
\hline Age (years) & 1.02 & $0.97-1.08$ & 0.477 & & & \\
\hline ALT level & 1.11 & $0.41-2.99$ & 0.846 & & & \\
\hline HBV DNA & 1.29 & $0.97-1.72$ & 0.083 & 1.44 & $1.02-2.03$ & 0.041 \\
\hline BMI & 1.06 & $0.92-1.23$ & 0.417 & & & \\
\hline AST levels & 6.55 & $1.44-29.84$ & 0.015 & 5.31 & $1.01-27.97$ & 0.049 \\
\hline
\end{tabular}

$\mathrm{B}, \mathrm{HBeAg}$ negative patients $(\mathrm{n}=167)$

Univariate analysis

Multivariate analysis

\begin{tabular}{|c|c|c|c|c|c|c|}
\hline Variables & OR & $95 \%$ CI & $\mathrm{P}$-value & OR & $95 \%$ CI & P-value \\
\hline Sex & 0.55 & $0.21-1.42$ & 0.217 & & & \\
\hline Age (years) & 1.01 & $0.95-1.06$ & 0.893 & & & \\
\hline ALT levels & 7.91 & $2.61-24.05$ & $<0.001$ & 5.42 & $1.74-16.910$ & 0.004 \\
\hline HBV DNA & 1.41 & $1.05-1.89$ & 0.021 & 1.42 & $1.00-2.01$ & 0.05 \\
\hline BMI & 1.07 & $0.92-1.23$ & 0.378 & & & \\
\hline AST levels & 2.06 & $0.55-7.71$ & 0.284 & & & \\
\hline
\end{tabular}

OR, odds ratio; CI, confidence intervals; ALT, alanine aminotransferase; HBV, hepatitis B virus; AST, aspartate aminotransferase; BMI, body mass index; $\mathrm{HBe} \mathrm{Ag}$, hepatitis B e antigen.

for advanced liver fibrosis. Among patients that were $\mathrm{HBeAg}$ negative, ALT levels $(\mathrm{P}=0.004)$ and HBV DNA $(\mathrm{P}=0.050)$ were independent predictors for advanced liver fibrosis.

Evaluation of suitable cut-off value of ALT levels and $H B V$ DNA load as predictors. AUROC was used to examine the use of ALT levels and HBV DNA load as indicators for advanced liver fibrosis (METAVIR score F2-F4; Fig. 3). The AUROC was $0.65(\mathrm{P}=0.003)$ when using ALT levels to predict advanced liver fibrosis. When an ALT level $\geq 0.88$ ULN was set as a positive indicator for advanced liver fibrosis, sensitivity and specificity were 87.8 and $47.4 \%$, respectively. The AUROC was 0.64 when HBV DNA load was set as an indicator for advanced liver fibrosis $(\mathrm{P}=0.004)$. If an $\mathrm{HBV} \mathrm{DNA}$ value $\geq 4.99$ $\log _{10} \mathrm{IU} / \mathrm{ml}$ was set as positive for advanced liver fibrosis, the sensitivity and specificity were 78.0 and $49.5 \%$, respectively.

When ALT, HBV DNA load and sex were combined, a formulation was obtained as follows: $-2.632+(0.93 \times \mathrm{ALT})+(0.258 \times \mathrm{DNA})-(0.995 \mathrm{x} \mathrm{sex})($ male $=1$, female $=2)$. The formulation score was well correlated with LS ( $r=0.33, P<0.001$, data not shown). The AUROC was $0.72(\mathrm{P}<0.001)$ when using the formulation score to predict advanced liver fibrosis. When the formulation score was set at $>-2.22$, the sensitivity and specificity to predict advanced hepatic fibrosis were 61.5 and $70.7 \%$, respectively (Fig. 3).

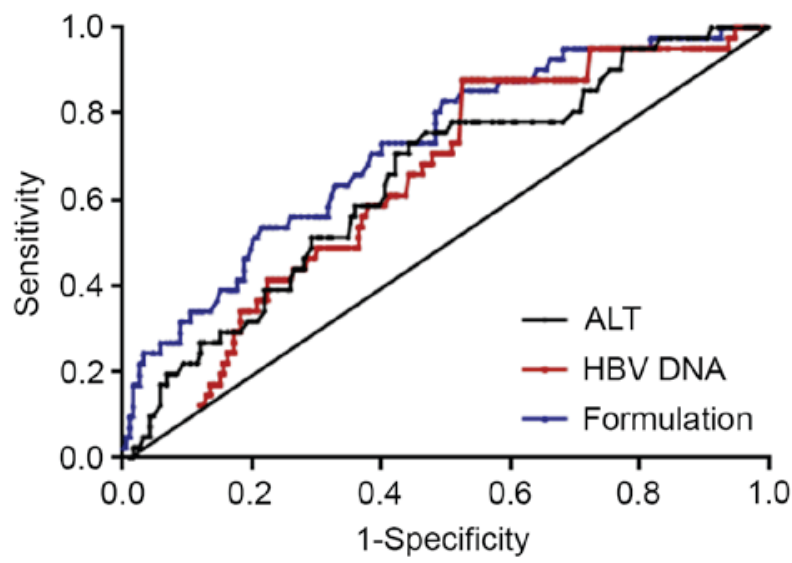

Figure 3. Analysis of AUROC for ALT levels, HBV DNA viral load and formulation score as indicators for advanced liver fibrosis. The AUROC for ALT was $0.65(\mathrm{P}=0.003)$, for HBV DNA viral load was $0.64(\mathrm{P}=0.004)$ and for the formulation score was $0.72(\mathrm{P}<0.001)$. AUROC, area under the receiving operator characteristic curve; $\mathrm{HBV}$, hepatitis B virus; $\mathrm{ALT}$, alanine aminotransferase.

\section{Discussion}

The results of the present study demonstrate that normal ALT levels do not always indicate the absence of advanced liver 
fibrosis, although ALT and HBV DNA levels are independent predictors of hepatic fibrosis in patients with $\mathrm{CHB}$ (16). The present data indicated that $54.1 \%$ (40/74) and $72.3 \%$ (115/159) of patients with chronic HBV infection with PNALT or PIEALT have liver fibrosis, respectively. These results are notable as clinical guidelines recommend that patients with liver fibrosis should not undergo treatment; consequently, these patients are often ignored. ALT levels are commonly used to assess liver inflammation and elevation of ALT is regarded as an indicator for anti-HBV treatment (5). However serum ALT levels may vary due to BMI, sex and metabolism disorder $(8,17,18)$. Therefore, ALT levels are an imperfect marker of the severity of liver diseases. According to the results of the present study, normal ALT levels do not indicate the absence of liver fibrosis in patients with HBV infection. A combined analysis of ALT levels, sex and serum HBV DNA load may more effectively identify high-risk patients with HBV infection. To the best of our knowledge, the study is the first to construct a formulation to identify advanced hepatic fibrosis among this population. However, the use of this formulation alone is not sufficient for clinical use. It would be more practical to combine Fibroscan with the formulation score to screen for liver fibrosis in patients with $\mathrm{CHB}$.

Chronic HBV infection exacerbates liver fibrosis in patients. Wang et al (19) reported that interleukin (IL)-17 expression increased in HBV-associated diseases, including CHB and post-hepatitis B liver cirrhosis. Furthermore, there was a positive correlation between IL-17 expression and the grade of liver fibrosis. Bai et al (20) reported that HBV Dane particles, $x$ protein and c protein may induce hepatic stellate cell proliferation via the platelet-derived growth factor (PDGF)-B/PDGF receptor- $\beta$ signaling pathway, which serves a key function in liver fibrosis caused by HBV infection.

In order to determine the antiviral treatment trigger point and improve survival rates, it is important to be able to rapidly identify patients with $\mathrm{CHB}$ at risk of developing fibrosis. Fagone et al (21) performed a meta-analysis of two datasets from two different rodent species and identified 254 and 132 genes that were significantly upregulated and downregulated, respectively. These biomarkers may provide novel pharmacological targets and be useful in assessing the progression of CHB. Combined antiviral treatment and novel therapeutic approaches may be effective at preventing end-stage liver diseases, such as liver cirrhosis (22).

Another potential diagnostic method of assessing liver fibrosis may be the analysis of circulating microRNAs (miRNAs). miRNAs are short RNA sequences 22 nucleotides long that post-transcriptionally regulate gene expression. Zhang et al (23) reported that a number of miRNAs were differentially expressed in patients with $\mathrm{CHB}$ and liver fibrosis. The miRNAs identified were involved in 100 signal transduction pathways, the majority of which affected liver fibrosis via the transforming growth factor- $\beta /$ Smad, Wnt, mitogen-activated protein kinase, Janus kinase/signal transducers and activators of transcription and vascular endothelial growth factor pathways. A study conducted by Lambrecht et al (24) reported that miRNA-200b and miRNA-122 were significantly upregulated during early liver fibrosis and that miRNA-192, $-92 \mathrm{a}$ and -150 were significantly downregulated in patients with HBV. Zheng et al (25) identified that levels of serum
miR-125a-5p correlated with liver fibrosis and suggested that serum miR-125a-5p may be used as a non-invasive biomarker to monitor the progression of liver disease.

Previous studies have demonstrated that advanced liver fibrosis is a precursor to end-stage liver diseases, including HCC (26). Chronic HBV infection with serum HBeAg-negative and serum HBV DNA-positive is commonly encountered in clinical practice. A proportion of patients with PNALT or PIEALT will develop advanced liver fibrosis (27) and it has been suggested that such patients should be closely monitored with TE or liver biopsy (28). Although liver biopsy is still regarded as the gold standard for assessing liver fibrosis, the invasive nature of the procedure results in complications arising in $0.5 \%$ of cases and the mortality rate of liver biopsy is $\sim 0.05 \%$. Sampling error and inter-observer variability may also lead to the understaging of cirrhosis (5). Therefore, it is important to develop novel non-invasive methods for fibrosis detection. Fibroscan is a non-invasive and reliable method of examining the degree of hepatic fibrosis. Since its detection range is 100 times greater than that of a liver biopsy, Fibroscan may more accurately reflect the degree of liver fibrosis (29). However, the diagnostic accuracy of Fibroscan may be affected by a number of factors, including elevated ALT levels and high BMI values (29). In addition, due to the difficulty of operation, cost and lack of skilled operators, Fibroscan is not yet widely used to determine LS. In the present study, the progression of liver fibrosis in patients with PNALT or PIEALT was evaluated according to sex, HBV DNA viral load and ALT levels. It was indicated that male patients with ALT in normal high levels (0.5 ULN $\leq$ ALT level<ULN) and high HBV DNA viral load are at a higher risk of developing advanced liver fibrosis, suggesting that liver biopsy should be considered for such patients.

In the present study, the proportion of patients with liver fibrosis in the PIEALT group was significantly higher compared with the PNALT group. ALT may be released into the circulation when hepatocytes are damaged. ALT fluctuations in the PIEALT group indicate that inflammation of the liver in these patients is more severe compared with the PNALT group. Repeated inflammatory activity in the liver will result in a more severe degree of liver fibrosis (28). A previous study with long-term follow-up indicated that patients with elevated ALT have a higher mortality rate (30). According to the present study, liver disease may progress in patients with PNALT, particularly those with normal-high ALT levels. A new ALT ULN value (male, $30 \mathrm{U} / 1$, female, $19 \mathrm{U} / 1$ ) has been proposed (31). Lin et al (32) reported that HBeAg-negative patients with high-normal ALT and elevated HBV DNA levels were more likely to experience adverse long-term outcomes. High-normal ALT was considered to be associated with liver injury and the revised ULN of ALT may be more suitable than the previous ULN (40 U/l) for screening patients with $\mathrm{CHB}$ to determine disease progression. The use of antiviral treatment and liver biopsy remains controversial for patients with CHB that have ALT levels below the current definition of ALT as $<2 x$ ULN but higher than the new ULN definition.

In the present study, being male was identified as one of the independent indicators of advanced liver fibrosis. Similar results have been observed in the healthy population (33). 
One hypothesis is that ovarian hormones may reduce expression of types I and III procollagen and inhibit deposition of hepatic collagen proteins, thereby inhibiting development of fibrosis (34). According to the results of the current study, sex should be taken into account when diagnosing advanced hepatic fibrosis. The current results also highlight concerns about the current guidelines that recommend using only ALT, HBsAg and HBV DNA levels to define different phases of HBV infection without performing liver biopsy (5). The current study suggests that patients with chronic HBV infection, PNALT and high HBV DNA viral load may progress to advanced liver disease. Further investigations are required to design a novel method of defining the progression of HBV infection.

Recently, Lemoine et al (35) reported that the $\gamma$-glutamyl-transpeptidase to platelet ratio (GPR) was a novel serum model able to accurately estimate advanced fibrosis and cirrhosis among patients with CHB in West Africa. GPR has several beneficial features, such as relatively high sensitivity and specificity, compared with other noninvasive methods. However, as the authors conclude, GPR requires further evaluation in other patient populations. AST to platelet ratio index (APRI) and GPR have previously been used to diagnose cirrhosis in patients with $\mathrm{CHB}(8,36)$. However, the two parameters have limitations, including a low level of sensitivity and positive predictive value, and a lack of evidence in patients with CHB and PNALT or PIEALT. In the present study, it was demonstrated that a combination of ALT levels, sex and serum HBV DNA load may more effectively identify patients with hepatitis at high risk of developing fibrosis, compared with ALT levels or HBV DNA load alone. The formulation has the advantage of only requiring inexpensive laboratory tests that are available in primary care centers; therefore it may be used to screen for liver fibrosis in resource-limited areas.

One limitation of the current study is that the results may be biased by the fact that all clinical data was collected from one research center. However, the sample size of the present study was sufficient to identify the predictors of liver fibrosis among patients with CHB and PNALT or PIEALT. To further validate the results, a prospective and multicenter study on a larger scale is warranted.

In conclusion, normal ALT levels do not always indicate the absence of hepatic fibrosis. A combination of ALT levels, sex and serum HBV DNA load may more effectively identify patients with $\mathrm{CHB}$ at high risk of developing fibrosis.

\section{References}

1. Clements CJ, Baoping Y, Crouch A, Hipgrave D, Mansoor O, Nelson CB, Treleaven S, van Konkelenberg R and Wiersma S: Progress in the control of hepatitis B infection in the Western Pacific Region. Vaccine 24: 1975-1982, 2006.

2. Liaw YF: Antiviral therapy of chronic hepatitis B: Opportunities and challenges in Asia. J Hepatol 51: 403-410, 2009.

3. Ott JJ, Stevens GA, Groeger J and Wiersma ST: Global epidemiology of hepatitis B virus infection: New estimates of age-specific HBsAg seroprevalence and endemicity. Vaccine 30: 2212-2219, 2012.

4. Liaw YF, Kao JH, Piratvisuth T, Chan HL, Chien RN, Liu CJ, Gane E, Locarnini S, Lim SG, Han KH, et al: Asian-Pacific consensus statement on the management of chronic hepatitis B A 2012 update. Hepatol Int 6: 531-561, 2012.
5. Sarin SK, Kumar M, Lau GK, Abbas Z, Chan HL, Chen CJ, Chen DS, Chen HL, Chen PJ, Chien RN, et al: Asian-Pacific clinical practice guidelines on the management of hepatitis B: A 2015 update. Hepatol Int 10: 1-98, 2016.

6. Rukunuzzaman M and Karim MB: Chronic hepatitis B in children - a review. Mymensingh Med J 24: 649-656, 2015.

7. Bravo AA, Sheth SG and Chopra S: Liver biopsy. N Engl J Med 344: 495-500, 2001.

8. Peng J, Cai S, Yu T, Chen Y, Zhu Y and Sun J: Aspartate aminotransferase to platelet ratio index-a reliable predictor of therapeutic efficacy and improvement of Ishak score in chronic hepatitis B patients treated with nucleoside analogues. Scand J Clin Lab Invest 76: 133-142, 2016.

9. Zeng J, Cai S, Liu J, Xue X, Wu X and Zheng C: Dynamic changes in liver stiffness measured by transient elastography predict clinical outcomes among patients with chronic hepatitis B. J Ultrasound Med 36: 261-268, 2017.

10. Cai S, Cao J, Yu T, Xia M and Peng J: Effectiveness of entecavir or telbivudine therapy in patients with chronic hepatitis B virus infection pre-treated with interferon compared with de novo therapy with entecavir and telbivudine. Medicine (Baltimore) 96: e7021, 2017.

11. European Association for the Study of Liver: EASL clinical practical guidelines: Management of alcoholic liver disease. J Hepatol 57: 399-420, 2012.

12. Cai SH, Lv FF, Zhang YH, Jiang YG and Peng J: Dynamic comparison between Daan real-time PCR and Cobas TaqMan for quantification of HBV DNA levels in patients with CHB. BMC Infect Dis 14: 85, 2014.

13. Castera L: Transient elastography and other noninvasive tests to assess hepatic fibrosis in patients with viral hepatitis. J Viral Hepat 16: 300-314, 2009.

14. Poynard T and Bedossa P: Age and platelet count: A simple index for predicting the presence of histological lesions in patients with antibodies to hepatitis $C$ virus. METAVIR and CLINIVIR Cooperative Study Groups. J Viral Hepat 4: 199-208, 1997.

15. Sandrin L, Fourquet B, Hasquenoph JM, Yon S, Fournier C, Mal F, Christidis C, Ziol M, Poulet B, Kazemi F, et al: Transient elastography: A new noninvasive method for assessment of hepatic fibrosis. Ultrasound Med Biol 29: 1705-1713, 2003.

16. Cai S, Yu T, Jiang Y, Zhang Y, Lv F and Peng J: Comparison of entecavir monotherapy and de novo lamivudine and adefovir combination therapy in $\mathrm{HBeAg}$-positive chronic hepatitis B with high viral load: 48-week result. Clin Exp Med 16: 429-436, 2016.

17. Sharaiha RZ, Kumta NA, Saumoy M, Desai AP, Sarkisian AM, Benevenuto A, Tyberg A, Kumar R, Igel L, Verna EC, et al: Endoscopic sleeve gastroplasty significantly reduces body mass index and metabolic complications in obese patients. Clin Gastroenterol Hepatol 15: 504-510, 2017.

18. Ou H, Cai S, Liu Y, Xia M and Peng J: A noninvasive diagnostic model to assess nonalcoholic hepatic steatosis in patients with chronic hepatitis B. Therap Adv Gastroenterol 10: 207-217, 2017.

19. Wang L, Chen S and Xu K: IL-17 expression is correlated with hepatitis B-related liver diseases and fibrosis. Int J Mol Med 27: 385-392, 2011.

20. Bai Q, An J, Wu X, You H, Ma H, Liu T, Gao N and Jia J: HBV promotes the proliferation of hepatic stellate cells via the PDGF-B/PDGFR- $\beta$ signaling pathway in vitro. Int J Mol Med 30: 1443-1450, 2012.

21. Fagone P, Mangano K, Mammana S, Pesce A, Pesce A, Caltabiano R, Giorlandino A, Portale TR, Cavalli E, et al: Identification of novel targets for the diagnosis and treatment of liver fibrosis. Int J Mol Med 36: 747-752, 2015.

22. Fagone P, Mangano K, Pesce A, Portale TR, Puleo S and Nicoletti F: Emerging therapeutic targets for the treatment of hepatic fibrosis. Drug Discov Today 21: 369-375, 2016.

23. Zhang Q, Xu M, Qu Y, Li Z, Zhang Q, Cai X and Lu L: Analysis of the differential expression of circulating microRNAs during the progression of hepatic fibrosis in patients with chronic hepatitis B virus infection. Mol Med Rep 12: 5647-5654, 2015.

24. Lambrecht J, Jan Poortmans P, Verhulst S, Reynaert H, Mannaerts I and van Grunsven LA: Circulating ECV-associated miRNAs as potential clinical biomarkers in early stage HBV and HCV induced liver fibrosis. Front Pharmacol 8: 56, 2017.

25. Zheng J, Zhou Z, Xu Z, Li G, Dong P, Chen Z, Lin D, Chen B and Yu F: Serum microRNA-125a-5p, a useful biomarker in liver diseases, correlates with disease progression. Mol Med Rep 12: 1584-1590, 2015 
26. Yuen MF, Yuan HJ, Wong DK, Yuen JC, Wong WM, Chan AO, Wong BC, Lai KC and Lai CL: Prognostic determinants for chronic hepatitis B in Asians: Therapeutic implications. Gut 54: 1610-1615, 2005

27. Kumar M, Sarin SK, Hissar S, Pande C, Sakhuja P, Sharma BC, Chauhan R and Bose S: Virologic and histologic features of chronic hepatitis B virus-infected asymptomatic patients with persistently normal ALT. Gastroenterology 134: 1376-1384, 2008.

28. Terrault NA, Bzowej NH, Chang KM, Hwang JP, Jonas MM and Murad MH; American Association for the Study of Liver Diseases: AASLD guidelines for treatment of chronic hepatitis B. Hepatology 63: 261-283, 2016.

29. Xue $X$ and Cai S: Comment on 'assessment of liver stiffness in pediatric fontan patients using transient elastography'. Can J Gastroenterol Hepatol 2016: 9343960, 2016.

30. Tai DI, Lin SM, Sheen IS, Chu CM, Lin DY and Liaw YF: Long-term outcome of hepatitis B e antigen-negative hepatitis B surface antigen carriers in relation to changes of alanine aminotransferase levels over time. Hepatology 49: 1859-1867, 2009.

31. Prati D, Taioli E, Zanella A, Della Torre E, Butelli S, Del Vecchio E, Vianello L, Zanuso F, Mozzi F, Milani S, et al: Updated definitions of healthy ranges for serum alanine aminotransferase levels. Ann Intern Med 137: 1-10, 2002.
32. Lin CL, Liao LY, Liu CJ, Yu MW, Chen PJ, Lai MY, Chen DS and Kao JH: Hepatitis B viral factors in $\mathrm{HBeAg}$-negative carriers with persistently normal serum alanine aminotransferase levels. Hepatology 45: 1193-1198, 2007.

33. Kumar M, Sharma P, Garg H, Kumar R, Bhatia V and Sarin SK: Transient elastographic evaluation in adult subjects without overt liver disease: Influence of alanine aminotransferase levels. J Gastroenterol Hepatol 26: 1318-1325, 2011.

34. Yasuda M, Shimizu I, Shiba M and Ito S: Suppressive effects of estradiol on dimethylnitrosamine-induced fibrosis of the liver in rats. Hepatology 29: 719-727, 1999.

35. Lemoine M, Thursz M, Mallet V and Shimakawa Y: Diagnostic accuracy of the gamma-glutamyl transpeptidase to platelet ratio (GPR) using transient elastography as a reference. Gut 66: 195-196, 2017.

36. Li Q, Lu C, Li W, Huang Y and Chen L: The independent predictors of significant liver histological changes in chronic hepatitis $B$ virus infection patients with persistently high-normal or low-normal alanine transaminase levels. Discov Med 23: 19-25, 2017. 